\title{
Olfaction as Sensors and Actuators
}

\author{
Hyung Gi Byun ${ }^{1}$, Chong Yun Kang ${ }^{2}$, Hae Ryong Lee ${ }^{3}$ \\ 1 Div. of Electronics, Information \& Communication Eng. Kangwon National university, Korea \\ ${ }^{2}$ Center for Electronic Materials, KIST, Korea \\ ${ }^{3} S W$ \& Contents Research Laboratory, ETRI, Korea \\ byun@kangwon.ac.kr
}

\begin{abstract}
Functionality of sensors and actuators in smart portable devices plays an important role in corresponding to five senses of human, enhancing user convenience, and enriching user experience. The human sense of small (olfaction) is perhaps the most interesting and yet is understood the least. A human interface for olfaction is composed of an odor sensing and olfactory display system. There is considerable need for a system that could mimic the olfaction and to use for various applications as a system based on sensing and actuating. In this presentation, we introduce an odor sensing system for healthcare monitoring of diabetes using human exhaled breath and an olfactory display which display odors to human being applicable to multimedia as an actuating activity for olfaction.
\end{abstract}

Key words: Olfaction, Odor sensing system, Olfactory display, Healthcare, Multimedia application.

\section{Introduction}

The human sense of smell (olfaction) is perhaps the most interesting and yet is understanding. A human interface for olfaction is composed of an olfactory display using actuating purpose and an odor sensing system called an electronic nose as sensing purpose. An olfactory display may be an output of machine, where the odor sensing system is its inputs [1, 2]. An odor sensing system containing an array of chemical sensors with partial specificity and an appreciate engine for recognition based on machine learning. It is able to recognize simple and complex gases and/or odors from various sources. Following by rapid developing for sensors' material and ICT technologies, olfaction sensing device is one of the strong candidates for loT applicable to environmental monitoring and healthcare as a portable device with small size, low power, and user friendly. Since 90's computer controllable olfactory display have been introduced as a scent generator for various odors. Recently, people started to concern about olfactory display using a device which displays odor to human being according to moving pictures for loT applicable to multimedia. Active researches in odor transmission and scent generating device are progressed. We have been developing an odor sensing system for healthcare monitoring of diabetes using human breath samples. The system has been constructed by metal oxide sensors array based on nanotechnology and a chamber can be used for SPME (Solid Phase Micro Extraction). In addition, we developing an olfactory display using compute controlled scent generators applicable multimedia. In this presentation, we introduce an odor sensing system applicable to exhaled breath analysis for diabetics and olfactory display applicable moving picture and virtual reality as case studies.

\section{Odor Sensing System for Breath Analysis}

The sense of smell used to be a common diagnostic tool in medicine, and physicians to use their sense of smell during their medical training. We are developing an intelligent odor sensing system for non-invasive healthcare monitoring combine a laboratory based chemical sensors module, pattern recognition subsystem and non-invasive sampling of volatile emitted from a patient into a highly intelligent sensor system that allows the rapid processing of these samples. It is capable to assist early and rapid diagnosis of changes in state of patient, and aid decision making by medical personnel in the treatment of such patients [3]. The odor sensing system consists of metal oxide type chemical sensor array with SPME fiber to detect low concentration of VOC 
(Volatile Organic Compounds) in the exhaled breath has been applied for monitoring diabetics. We collected exhaled breath samples from diabetic patients and healthy subjects to determine whether samples can be differentiated from both groups on the basis of VOCs. Fig. 1 is shown the primary result for classification between healthy and diabetics group.

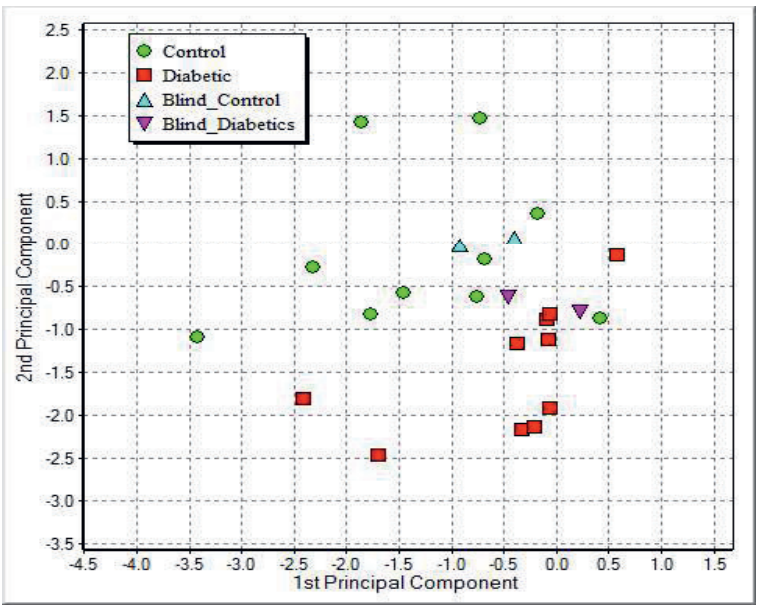

Fig. 1. The primary result of PCA plot for healthy and diabetics group

\section{Olfactory Display for Multimedia Application}

Until now most users have employed three kinds of sensory perception for human interface. Typically user interfaces for visual display are texts, graphics, and animation for auditory purpose speech or non-speech sounds are used; and for haptics, vibration amplitude and frequency change feedback from a haptic device are used. In the case of olfaction, there are a little concern regarding the olfactory display. Recently, computer controllable olfactory display system have been developed and applied various applications such as scent alarm, game, education, and entertainment including 4-D Multiplex. We demonstrate an olfactory display applicable moving picture and virtual reality. The scent generator consisted ultra vibrator and odor cartridge is developed to deliver odors controlled by odor blending and display software shown in Fig. 2.

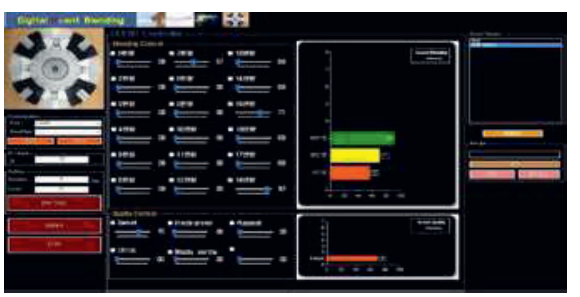

Fig. 2. The scent generator and controlled software for odor blending and display
We applied the olfactory display to virtual reality with HMD shown in Fig.3

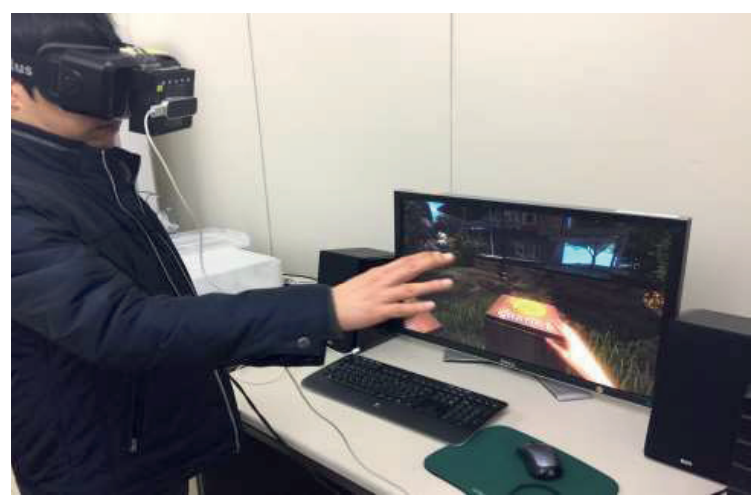

Fig. 2. The Olfactory display for VR Application

\section{Summary}

In this presentation, we demonstrated an olfaction sensing system for healthcare monitoring system based on chemical sensors array to exhale breath analysis and olfaction actuating system for multimedia application based olfactory display separately. In case of olfaction, an olfactory display is an output from machine, whereas an odor sensing system is its input. In machine olfaction, odor information obtained from an odor sensing system is transmitted to an olfactory display via the network following by standard protocol. We need more comprehensive research works for the next generation olfaction.

\section{References}

[1] T. Nakamoto (Ed.) Human olfactory displays and interface, Hershey, PA: IGI-Global, 2013

[2] T. Nakamoto (Ed.) Essentials of Machine Olfaction and Taste, Willy, 2016

[3] H. Byun, J. Yu, J. Jeon, S. Lee, J. Huh, J. Lim Exhaled Breath Analysis for Lung Diseases Based on Sensor Array System, Sensor Letters 12, 1-3 (2014)

\section{Acknowledgements}

This work was supported by Institute for Information \& Communication Technology Promotion (IITP) grant funded by the Korea Government (MSIP) (No. 2015-0-00318, Olfactory Bio Data based Emotion Enhancement Interactive Content Technology Development). 